\title{
Optimizing Workflow Scheduling using Max-Min Algorithm in Cloud Environment
}

\author{
Sandeep Singh Brar \\ ME Scholar - CSE \\ Chandigarh University \\ Gharuan, Mohali
}

\author{
Sanjeev Rao \\ Assistant Professor \\ Chandigarh University \\ Gharuan, Mohali
}

\begin{abstract}
With the up rise of fourth paradigm, that is discovery of science over a prolonged period of time, scientific workflows commence to amend their status amongst innumerous science subject areas including physics, astronomy, biology, chemistry, earthquake science and many more. In Scientific workflows, a heavy volume of data processing is required and workflows with up to a few million tasks are not unusual. With the advent of Cloud Computing as a new model of service provisioning in distributed systems, a new direction comes in light for executing scientific applications such as Workflows by deploying resources of Cloud. The scheduling of millions of tasks of workflows, while processing with Cloud resources, in a most profitable manner i.e. minimum computation time is still an attractive research area. The existing scheduling algorithms are brushing off the individual dependent and independent tasks. In this research paper, MaxMin algorithm is implemented for scheduling of workflow tasks that is focalized on the consideration of dependent and independent tasks and process independent tasks in parallel that directly gives profit in minimizing computation time.
\end{abstract}

\section{General Terms}

Cloud Computing, Scheduling Algorithms.

\section{Keywords}

Cloud Computing, Cloud broker, Scheduling Algorithms, Workflow, Scheduling.

\section{INTRODUCTION}

When you store your photos on-line rather than on your data processor, utilize web emails or social networking websites, you are employing a service of Cloud Computing. Rapid change has been seen in the field of dispersed Computing with time. Initially, desktop computing, then grid computing and finally Cloud Computing took business into a new direction because of unlimited features contributed by it. The essence accusative of variation in technology is to provide best computing facilities to unlimited end-users in reliable, efficient, securable and scalable manner. All these technologies extend the features of internet and based on utility computing. Cloud Computing concerns with the availability of resources that can help in computing at the web, rather than confined on your own drive or change applications according to own wishes, you employ a service on the web, at an additional place, to stock up your data or utilize its applications.

Cloud Computing opens new ways for numerous applications from which processing of scientific workflows is most crucial. The scientific workflows like Montage, Cyber Shake, Sipht etc. requires heavy processing of data that is only possible with the deployment of Cloud Computing resources. However, the optimized scheduling of the individual tasks of workflow is still an issue yet to solve. The numerous algorithms are proposed and implemented such as First Come First Serve, Data-aware algorithm, Min-Min algorithm, Round-Robin algorithm etc., but these are unable to consider the independent and dependent tasks individually and schedule the tasks in such a way that the independent tasks can process simultaneously. In this research paper, Max-Min algorithm is implemented that gives priority to the largest task and also process other independent simultaneously. This concept of Max-Min algorithm directly benefits in minimizing computation time. The paper is organized as follows. Section II describes the review of the literature work. Section III illustrates the methodology adopted in detail, and in Section IV results are evaluated. Lastly, in Section V conclusions are drawn and directions for future research are considered.

\subsection{Scheduling}

When a computer is running more than one processes that competes with one another for the CPU resources simultaneously, then there is a need to make a decision by Operating System that which process to run next. The process of taking decision that which process will get resources is called Scheduling. The element of Operating System which makes a choice about the next process is called Scheduler. The algorithm that is used for performing scheduling is regarded as Scheduling algorithm. As a vital part of Cloud Computing, task scheduling is a mechanism that helps in scheduling of subtasks of workflows by arranging the tasks in queue and providing them with the suitable resources that can execute these tasks. Cloud Computing has many peculiar features like virtualization and flexibility. With the help of technology of virtualization, all the resources that are physically available can be made virtualized and transparent for users. Additionally, more than one virtual machine are able to operate on a single host computer so that the rate of employment of resources has been effectively enhanced. Supplying resources under Cloud Computing environment is flexible; we increase or reduce the supplying of resources based on requirement of workflows.

\subsubsection{Need for Scheduling}

The primarily thing about transferring to utilizing Clouds is application quantifiability, This property of Cloud resources permits time period provisioning of resources to satisfy workflows prerequisites.

\subsubsection{Existing Scheduling Algorithms}

- First Come First Serve Algorithm: It is one of the most common algorithms that is employed in scheduling resources for different workflows. This algorithm works on a very simple principle that the 
request that comes first will be executed primarily. It does not consider the size of request neither account the dependency and independency of tasks that are requested.

- Round-Robin Algorithm: It is second most commonly used algorithm for assigning the resources, while scheduling, to the different requests submitted for execution. It is also implemented for utilizing Cloud Computing resources for different workflows. In this algorithm, the basic principle is that assign some predefined time slots to the submitted requests. It does not consider dependency and independency that exists between different tasks.

- Data-Aware Algorithm: It is well known algorithm for scheduling of Cloud Computing resources while executing different workflows. This algorithm works on a simple principle that can understand the size of submitted task and works like FCFS. It firstly execute the request that is firstly submitted to it.

- Min- Min Algorithm: It is one of the commonly known algorithm for scheduling of workflows. MinMin heuristic sets the priority to the task that requires the smallest execution time rather than the longest execution time. In each iterative step, choosing smallest executed process and expected to complete the task at the earliest time.

\subsection{Scientific Workflows}

With the up rise of fourth paradigm, that is discovery of science over a prolonged period of time, scientific workflows commence to amend their status amongst innumerous science subject areas including physics, astronomy, biology, chemistry, earthquake science and many more. In Scientific workflows, heavy volumes of data processing are required and workflows with up to a few million tasks are not unusual. Amongst these large-scale and loosely-coupled applications, the similar jobs within these requests are often fairly minor. However, critical amount of calculation and data is characterized by accumulation. The very best example of workflow is the Cyber Shake workflow that is employed by the Southern California Earthquake Center (SCEC) for machine parallel environment like Grid or the Cloud.

\section{Types of Workflows:}

Three wide used scientific workflows are: Montage, Cyber Shake and SIPHT.

1. Montage is an astronomy application that is used to build huge copy mosaics of the sky.

2. Cyber Shake is a geophysical science application that computes Probabilistic unstable Hazard curves for geographic sites in the Southern California region.

3. SIPHT workflow organize a good seek for minor untranslated RNAs that controls numerous procedures like secretion or virulence in bacteria.

\subsubsection{Demonstration of Workflow}

A workflow is conventionally demonstrated as Directed Acyclic Graph (DAG).

- $\quad$ Node of a DAG signifies a workflow task

- Edges signify dependency between the tasks ( $t$ ) that pressure the order in which the tasks are performed.
Every job includes a program and a set of factors that need to be carried out to fulfill the job. A job (j) itself is a single execution unit and it comprises of one or multiple tasks. The dependencies typically mean data flow dependencies in the application, because of result files yielded by one task are acquired as inputs for another task.

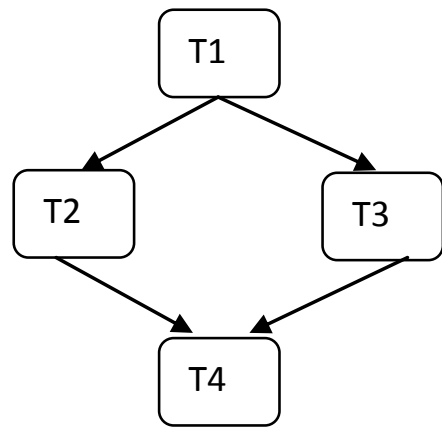

Fig. 1. Directed Acyclic Graph

\subsubsection{Challenges in Workflow Applications}

- Imbalance of Computation: Amongst the common challenge confronted because of merging workflow tasks is the imbalance of computation. Load imbalance is caused because of diversity of tasks at runtime.

- Data Organization within a Workflow: Scientific applications are often data modifiers for which a team of scientists is pre-requisite who belongs to various organizations, that results to distribution of requested data in scientific workflows. Primarily, with the blend of grids and clouds, scientists can upload their data and introduce their applications on scientific cloud workflow systems regardless of location through the Internet.

- Resource management: It is the third big challenge that comes forward with the combination of Cloud Computing and resource provisioning methods. Demand for different types of resources for execution has been increased rapidly with the increase of the amount of workflows. The potential to deliver resources ondemand is based on pay-per-use model and alter resource volume according to the varying demands of the application is offered by Infrastructure-as-a-Service (IaaS) clouds. Task clustering can still be implemented to this cloud situation. There is need to put attention and take decisions for cloud setup towards resource utilization factor along with the performance-related metrics because resources from commercial clouds generally have economic costs related with them.

- Fault Tolerance: Current clustering approaches overlook or undervalue the influence of incidence of failures on the behavior of systems, in spite of the growing influence of failures among the distributed systems that are scaled on a large basis. The value of fault acceptance design is highlighted by many researchers and they show that the rates of getting failed are important while considering the latest distributed systems. Temporary failures need more attention than the stable failures because they are predictable to be more widespread. 


\section{RELATED WORK}

\section{Rajkumar Buyya, Rajiv Ranjan, Rodrigo N. Calheiros [2009]}

Rajkumar Buyya et al. [1] introduce the simulation toolkit named as CloudSim that helps in simulating and modeling of environment of Cloud. With the help of this toolkit, desired number of virtual machines, datacenters etc. can be created. It enables the user to understand the concept of federation and migration of virtual machines for reliability of applications.

\section{K. Agrawal, A. Benoit, L. Magnan and Y. Robert [2010]}

$\mathrm{K}$. Agrawal et al. [2] presented the concept of scheduling and mapping of workflows in Cloud. Author arise the trouble of scheduling that comes in case of linear workflows if mapping is given. The scheduling algorithm can be used to lessen the retro or potential or both. Two models are used in arrangement, one is one-port model and another is multi-port model. In one port model all processes are sequential and restricted communication capacities and computation overlap comes in multi-port model.

Chen, R. M., Wu, C. L., Wang, C. M. and Lo, S. T. [2010] Chen et al. [3] presents a Particle swarm optimization (PSO) based approach that can be applied on the scheduling of projects where resources are constrained. The major issue in these projects is long duration of projects that is represented by the multidimensional particle. As a solution, two representations are offered that are priority-based representation and permutation-based representation. Then, frameworks are developed on the basis of two solution representations. In this paper, the outputs of these two representations are compared and a new option of developing a new mechanism that is useful for searching best particle from swarm is offered.

\section{Huang Q.Y., Huang T.L. [2010]}

Huang Q.Y. et al. [4] explains the importance of Cloud Computing that is supported by the virtualization technology. This concept is advertized by the business section rather than academic one due to its concentration on users applications. It arises the point that different clients have varied QoS prerequisites. This paper reviewed different scheduling models and compared them on the basis of given deadline and budget.

\section{Baomin Xu, Chunyan Zhao, Enzhao Hu, Bin Hu [2011]}

Baomin $\mathrm{Xu}$ et al. [5] proposed an algorithm that is purely based on Berger model. While implementing this algorithm to the scheduling process, it demonstrates the concept of fairness constraint. The basic constraint is to classify the tasks defined by users on the basis of QoS preferences and then develop a general expectation function fitting with classification of tasks.

\section{V.Krishna Reddy, B. Thirumala Rao , LSS Reddy [2011]}

V.Krishna Reddy et al. [6] gives the overview of emerging technique that has an enormous impact on the Information Technology (IT) within some past years. The author finds out the varied issues related with cloud computing and also search out interesting future research options. The different challenges of cloud computing are divided in four types such as security challenges, data challenges, performance challenges and design challenges.

\section{Li Jian Feng, Peng Jian [2011]}

Li Jian-feng et al. [7] described about the large number of users, tasks and data involved in cloud computing that arise a very important question that is how to schedule these tasks.
A new model Double-Fitness Genetic Algorithm(DFGA) is proposed for programming framework of cloud computing environment. This algorithm is able to not only shorten totaltask-completion time but also has shorter average-completion time. The result of DFGA is better than Adaptive Genetic Algorithm (AGA) when these two are compared through simulation experiment.

Jing Liu , Xing-Guo Luo, Xing-Ming Zhang3, Fan Zhang and Bai-Nan Li [2013]

Jing Liu et al. [8] proposes a new task scheduling model that is mainly focused on cut down the power consumption of Cloud Computing and amending the benefits of service providers. This algorithm is basically designed with the help of multi-objective genetic algorithm (MO-GA). This research consists of encoding rules, crossover operators, selection operators and the method of sorting Pareto solutions. With the help of CloudSim, this algorithm is implemented and compared with existing algorithms and it is successful in yielding better result for balancing the performance of multiple objects.

Tarun goyal , Aakanksha Agrawal [2013]

Tarun Goyal et al. [9] discusses the new paradigm of Cloud computing that helps the clients in employing the computation, storage, data and services throughout the globe in a commercial way. Among the most important issues of cloud computing, scheduling is still a striking research area. In this paper, a new scheduling model is offered that is centralized on the concept of Minimum Network Delay. In this model, Suffrage Heuristic is conjugate with genetic algorithms to schedule the independent tasks. This work is successful in reducing the make span.

\section{Swachil Patel, Upendra Bhoi [2013]}

Swachil Patel et al. [10] explained job scheduling priority as the biggest issue because some jobs need to schedule first than the other jobs that can wait for a long time. In this paper, a systematic review of various priority based job scheduling algorithms is presented. These algorithms have different perspectives and working principles.

\section{Rohit O. Gupta, Tushar Champaneria [2013]}

Rohit O. Gupta et al. [11] discusses the different kinds of scheduling algorithms that have been implemented for executing workflows. These algorithms are compared with each other on the basis of tools, scheduling factors and so on. This paper concludes that an algorithm is still demanded that is related with improvement of reliability and availability in Cloud environment.

\section{B. Anuradha, S. Rajasulochana [2013]}

B. Anuradha et al. [12] described the demand of scheduling in cloud computing environment due to the heterogeneous pool of resources. The scheduling is simply executing large number of jobs under given constraint. The concept of fairness in scheduling is mandatory criterion which improves the efficiency and provides optimal resource allocation.

\section{Jia Ru [2013]}

Jia Ru et al. [13] introduced a new and effective scheduling algorithm that is focused to maximize the cloud resource utilization, improve the computation ratio, reduce make span and overheads and delay in cloud based systems. The author reviewed all existing scheduling algorithms that has been implemented in Cloud Computing environment. 


\section{PROPOSED METHODOLOGY}

In our proposed work, Max-Min algorithm is proposed for optimizing workflow scheduling. In this approach, different types of workflows are assigned as a input. Then, apply different scheduling algorithms and execute. The results are obtained and analyzed on the basis of computation time and cost.

The proposed approach basically consists of:

1. Input: The Four wide used scientific workflows are involved as input : Montage, Cyber Shake, and SIPHT.

- Montage is an astronomy application that is used to build huge copy mosaics of the sky.

- Cyber Shake is a geophysical science application that computes Probabilistic unstable Hazard curves for geographic sites in the Southern California region.

- SIPHT workflow organize a good seek for minor untranslated RNAs (sRNAs) that controls numerous procedures like secretion or virulence in bacteria.

2. Allocate Resources: To all these workflows, basic resources are allocated.These resources include virtual machines, broker, cloudlets and datacenters.

\section{Apply Algorithms}

4. Compute Calculation Time

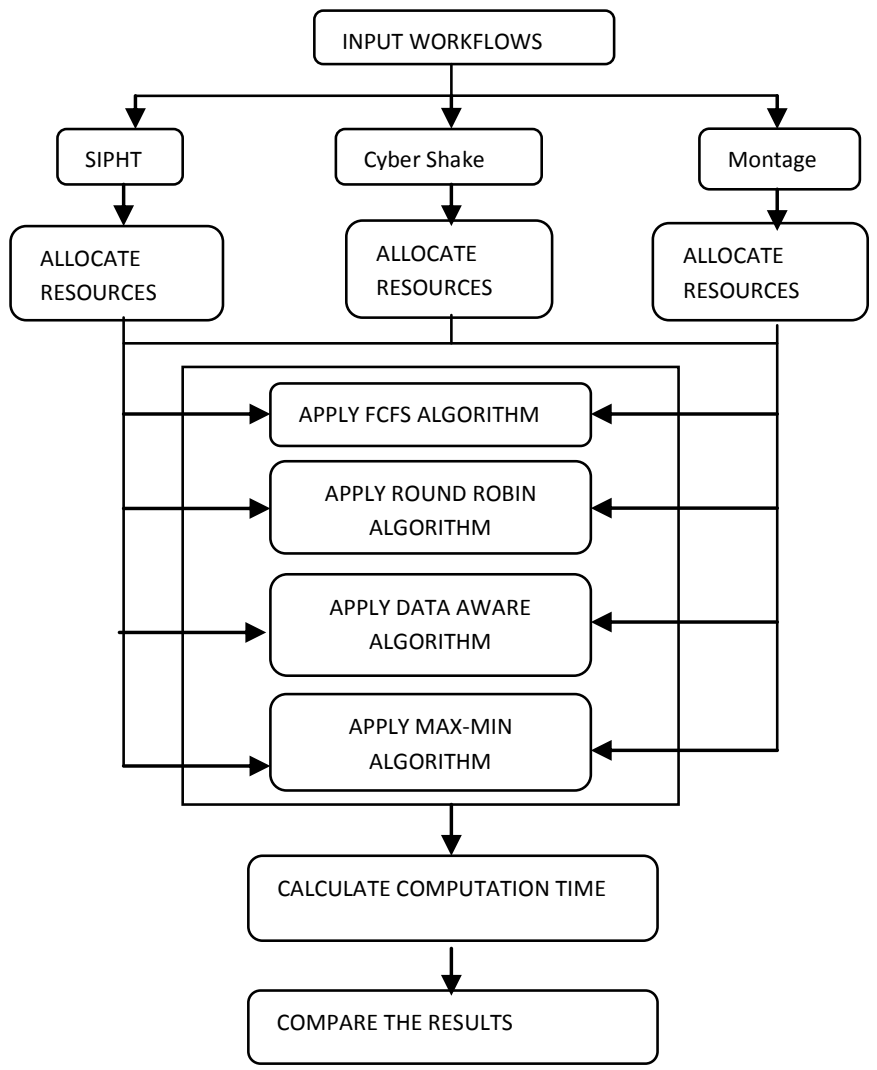

Fig. 2: Proposed Methodology

\subsection{Proposed Algorithm}

Input: Workflow of independent and dependent tasks

Output: Processed Workflows with Computation Time

1. For $\mathrm{I}=0$ to Length (workflow. tasks)

2. Begin

3. Separate tasks from according to flow of graph

4. Provide resources (virtual machine) to every task.

5. End

6. Apply Max-Min algorithm on Workflow

7. For $\mathrm{I}=0$ to Length (workflow .tasks)

8. Begin

9. Separate tasks from according to flow of graph

10. Provide resources according to Max-Min.

11. End

12. Analyze Computation Time

\section{IMPLEMENTATION AND RESULTS}

The output of Max- Min algorithm is compared with output of other popular algorithms such as First Come First Serve, Round Robin and Data-aware. These algorithms are executed on three popular workflows that are Sipht, Cyber Shake and Montage.

Table 1. Computation time when 100 nodes of SIPHT Workflow are processed

\begin{tabular}{|l|l|l|l|l|}
\hline Level & $\begin{array}{l}\text { First Come } \\
\text { First Serve } \\
\text { Algorithm }\end{array}$ & $\begin{array}{l}\text { Round } \\
\text { Robin } \\
\text { Algorithm }\end{array}$ & $\begin{array}{l}\text { Data-Aware } \\
\text { Algorithm }\end{array}$ & $\begin{array}{l}\text { Max-Min } \\
\text { Algorithm }\end{array}$ \\
\hline 0 & 1.1 & 0.2 & 0.1 & 0.11 \\
\hline 1 & 1885.97 & 1063.77 & 11427.34 & 6560.13 \\
\hline 2 & 1501.991 & 1947.99 & 1624.27 & 2023.76 \\
\hline 3 & 195.72 & 49.41 & 041.12 & 30.28 \\
\hline 4 & 1.36 & 0.34 & 80.52 & 0.35 \\
\hline
\end{tabular}

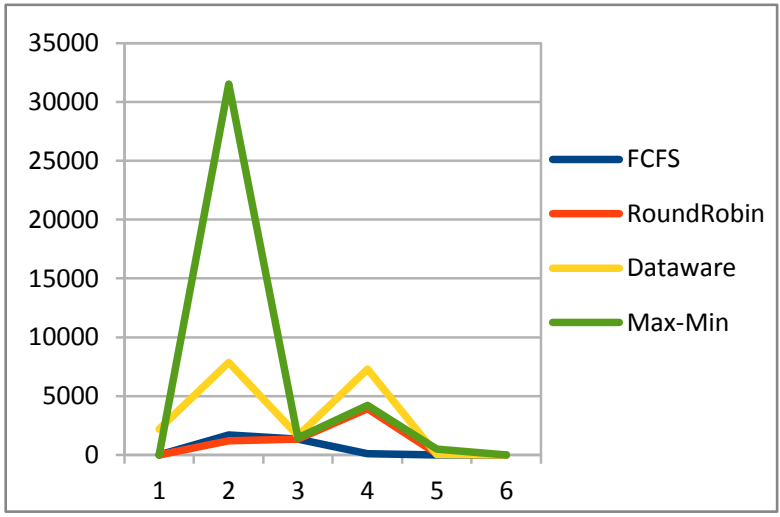

Fig. 3: Diagrammatically shown computation time for Sipht when scheduled by different algorithms 
Table 2. Computation time when 100 nodes of

CyberShake Workflow are processed

\begin{tabular}{|l|l|l|l|l|}
\hline Level & $\begin{array}{l}\text { First Come } \\
\text { First Serve } \\
\text { Algorithm }\end{array}$ & $\begin{array}{l}\text { Round } \\
\text { robin } \\
\text { Algorithm }\end{array}$ & $\begin{array}{l}\text { Data-Aware } \\
\text { Algorithm }\end{array}$ & $\begin{array}{l}\text { Max-Min } \\
\text { Algorithm }\end{array}$ \\
\hline 0 & 1.1 & 0.2 & 0.1 & 0.11 \\
\hline 1 & 4885.62 & 3063.55 & 11427.34 & 4560.13 \\
\hline 2 & 1501.99 & 3009.15 & 1624.27 & 1023.15 \\
\hline 3 & 132.15 & 49.41 & 5041.12 & 30.28 \\
\hline 4 & 1.36 & 0.34 & 80.52 & 0.35 \\
\hline
\end{tabular}

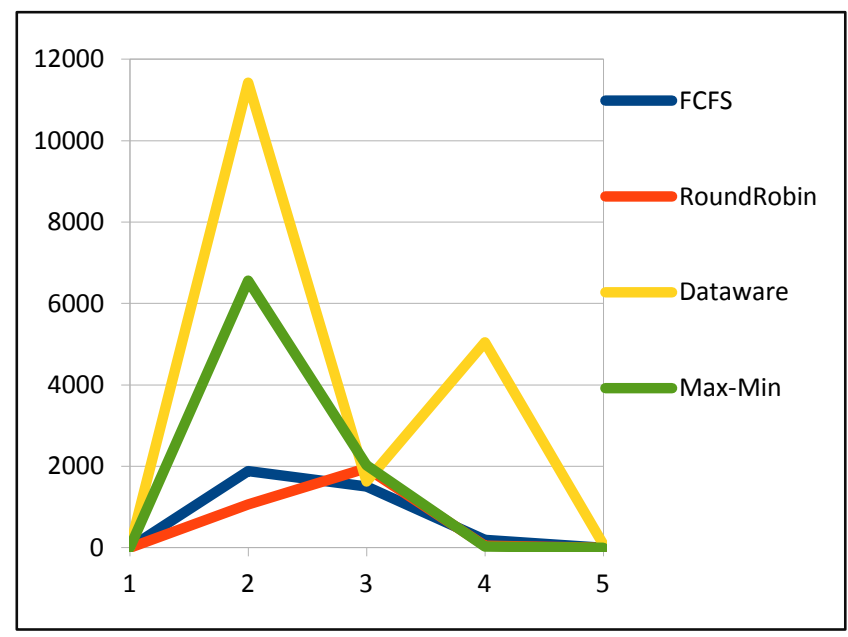

Fig. 4. Diagrammatically shown computation time for Cyber Shake when scheduled by different algorithms

Table 3. Computation time when 100 nodes of Montage Workflow are processed

\begin{tabular}{|c|c|c|c|c|}
\hline Level & $\begin{array}{l}\text { First Come } \\
\text { First Serve } \\
\text { Algorithm }\end{array}$ & $\begin{array}{l}\text { Round } \\
\text { robin } \\
\text { Algorithm }\end{array}$ & $\begin{array}{l}\text { Data- } \\
\text { Aware } \\
\text { Algorithm }\end{array}$ & $\begin{array}{l}\text { Max-Min } \\
\text { Algorithm }\end{array}$ \\
\hline 0 & 0.41 & 0.22 & 0.22 & 0.21 \\
\hline 1 & 110.7 & 215.6 & 96.39 & 154.48 \\
\hline 2 & 1348.7 & 860.24 & 330.24 & 321.23 \\
\hline 3 & 18.55 & 4.96 & 23.67 & 20.34 \\
\hline 4 & 34.23 & 34.67 & 6.49 & 5.36 \\
\hline 5 & 714.23 & 172.24 & 129.34 & 42.32 \\
\hline 6 & 36.42 & 7.63 & 11.35 & 8.55 \\
\hline 7 & 35.23 & 9.64 & 20.11 & 20.34 \\
\hline 8 & 25.85 & 6.91 & 8.39 & 6.94 \\
\hline 9 & 3.1 & 0.83 & 0.45 & 0.83 \\
\hline
\end{tabular}

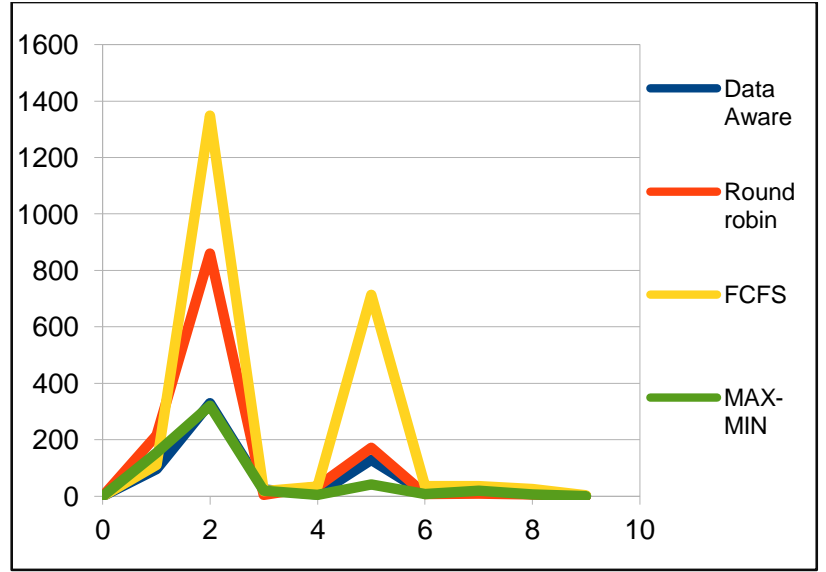

Fig. 5. Diagrammatically shown computation time for Montage when scheduled by different algorithms

Different algorithms like FCFS, Cyber Shake, Sipht and MaxMin are implemented for the scheduling of workflow processing. The results are compared on the basis of computation time. It is concluded that Max- Min yields best results for Montage as compare to other all algorithms. MaxMin yields good results for Cyber Shake as compare to Dataaware but unable to optimize the results as compare to FCFS and Round robin. Max-Min yields negative results for Sipht when compare to all other algorithms. . The reason for these variation among different workflows is that Montage consists of large number of large sized tasks, so Max-Min optimize its computation time, but Cyber Shake consists average number of large sized tasks, therefore its computation times is not optimized to a very good level. Lastly, workflow Sipht does not show any optimization in results due to the fact because it consists of maximum number of small tasks.

\section{CONCLUSION}

Scheduling is one of the most crucial jobs in cloud computing environment. We have implemented Max-Min algorithm which has focused to schedule multiple tasks in such a way that large sized task is firstly executed and simultaneously other independent tasks are also executed. The algorithm is able to schedule multiple jobs on multiple machines in an efficient manner such that the jobs take the minimum time for the completion. The algorithm is compared with existing algorithms and improves the execution time. Processing of different workflows with Cloud Computing offers a number of future research options. There is need to develop a algorithm on the basis of hybrid approach of Max- Min and Min-Min algorithm that can iteratively check size of nodes at each level and then execute suitable algorithm. The different metrics for parameters like cost, energy etc. can be also developed.

\section{REFERENCES}

[1] Rajkumar Buyya, Rajiv Ranjan, Rodrigo N. Calheiros, "Modeling and Simulation of Scalable Cloud Computing Environments and the CloudSim Toolkit: Challenges and Opportunities," The International Conference on High Performance Computing and Simulation, HPCS2009, pp:1-11, Year 2009.

[2] K. Agrawal, A. Benoit, L. Magnan and Y. Robert, "Scheduling Algorithms for Linear Workflow optimization," IEEE, Year 2010. 
[3] Chen, R. M., Wu, C. L., Wang, C. M. and Lo, S. T., "Particle swarm optimization scheme to solve resourceconstrained scheduling problem," Expert systems with applications, Vol. 37, pp. 1899-1910, ISSN: 0957-4174 March, Year 2010.

[4] Huang Q.Y., Huang T.L. , "An Optimistic Job Scheduling Strategy based on QoS for Cloud Computing , IEEE International Conference on Intelligent Computing and Integrated Systems, Guilin, pp. 673-675, Year 2010.

[5] Baomin Xu, Chunyan Zhao, Enzhao Hu, Bin Hu, "Job Scheduling algorithm using Berger model in Cloud Environment," Elsevier in Advances in Engineering Software, Vol. 42 , Issue No. 7, pp. 419-425, Year 2011.

[6] V.Krishna Reddy, B. Thirumala Rao , LSS Reddy, "Research issues in Cloud Computing," Global Journal Computer Science \& Technology, Vol. 11, pp.70-76, June, Year 2011

[7] Li Jian Feng, Peng Jian, "Task scheduling algorithm based on improved genetic algorithm in cloud computing environment," Journal of Computer Applications, pp 184-186, Year 2011.

[8] Jing Liu , Xing-Guo Luo, Xing-Ming Zhang3, Fan Zhang and Bai-Nan Li, "Job Scheduling Model for
Cloud Computing Based on Multi- Objective Genetic Algorithm," International Journal of Computer Science Issues, Vol. 10, Issue 1, ISSN : 1694-0784, January, Year 2013.

[9] Tarun goyal , Aakanksha Agrawal, "Host scheduling algorithm using genetic algorithm in cloud computing environment," International Journal of Research in Engineering and Technology, Vol. 1, Issue 1, pp. 7-12, June, Year 2013

[10] Swachil Patel, Upendra Bhoi, "Priority Based Job Scheduling Techniques In Cloud Computing," International Journal of Scientific \& Technology Research, Vol. 2, Issue 11, ISSN : 2277-861, November, Year 2013.

[11] Rohit O. Gupta, Tushar Champaneria, "A Survey of Proposed Job Scheduling Algorithms in Cloud Computing Environment," International Journal of Scientific \& Technology Research, Volume 3, Issue 11, November, Year 2013.

[12] B. Anuradha, S. Rajasulochana, "Fairness As Justice Evaluator In Scheduling Cloud Resources: A Survey," International Journal of Computer Engineering \& Science, ISSN: 22316590 , November, Year2013.

[13] Jia $\mathrm{Ru}$, "An Investigation on scheduling policies for cloud based software-systems". 